\title{
Identification of key pathways and genes in Barrett's esophagus using integrated bioinformatics methods
}

\author{
CONG ZHANG $^{1}$, YUJIE SHEN ${ }^{1}$, JIAZHENG WANG ${ }^{1}$, MINGXIA ZHOU $^{1}$ and YINGWEI CHEN $^{1,2}$ \\ ${ }^{1}$ Department of Gastroenterology, Xin Hua Hospital Affiliated to \\ Shanghai Jiao Tong University School of Medicine; ${ }^{2}$ Shanghai Key Laboratory of Pediatric Gastroenterology \\ and Nutrition, Shanghai Institute for Pediatric Research, Shanghai 200092, P.R. China
}

Received January 5, 2017; Accepted November 14, 2017

DOI: $10.3892 / \mathrm{mmr} .2017 .8274$

\begin{abstract}
Barrett's esophagus (BE) is a premalignant lesion of esophageal adenocarcinoma. The aim of the present study was to investigate the possible mechanisms and biomarkers of BE. To identify the differentially expressed microRNAs (DEmiRNAs) and genes (DEGs) in BE, the miRNA expression profile GSE20099 and the gene expression profiles GSE26886, GSE13083 and GSE34619 were obtained from the Gene Expression Omnibus (GEO) database. DEGs and DEmiRNAs were screened for using the GEO2R tool. Using DAVID, functional and pathway enrichment analysis was performed to explore the biological function of identified DEGs. The protein-protein interaction (PPI) network was detected using STRING and constructed by Cytoscape software. Furthermore, targets of identified DEmiRNAs were predicted by the miRecords database, then integrated with the identified DEGs to obtain key genes involved in BE. In total, 311 DEGs were identified. These genes were significantly enriched in the pancreatic secretion, metabolic pathways and cytochrome P450 drug metabolism pathways. In the PPI network, 16 hub genes, including keratin 16 , cystic fibrosis transmembrane conductance regulator, involucrin, protein kinase $\mathrm{C} \alpha$ and cadherin 17 were identified. Following integration of the predicted target genes of DEmiRNAs with DEGs, three key BE genes were identified: PRKCA, CDH17 and epiregulin. In conclusion, a comprehensive bioinformatics analysis of identified DEGs and DEmiRNAs was performed to elucidate potential pathways and biomarkers involved in the development of BE.
\end{abstract}

Correspondence to: Dr Yingwei Chen, Department of Gastroenterology, Xin Hua Hospital Affiliated to Shanghai Jiao Tong University School of Medicine, 1665 Kongjiang Road, Shanghai 200092, P.R. China

E-mail: chenyingwei@xinhuamed.com.cn

Key words: Barrett's esophagus, bioinformatics analysis, differentially expressed gene, differentially expressed microRNAs, functional enrichment analysis

\section{Introduction}

Barrett's esophagus (BE) is defined as a metaplasia of the lower esophagus, where the normal squamous epithelium is replaced by intestinalized columnar epithelium in response to gastroesophageal reflux disease (GERD). Population based studies estimate that the prevalence of BE is increasing, with the current incidence at $1.3-1.6 \%$ in general population of Sweden and Italy (1). Notably, BE is a premalignant lesion of esophageal adenocarcinoma (EAC) and significantly enhances the risk of EAC development (2). However, the molecular mechanism of BE development is still largely unknown.

A number of studies focused on the development of $\mathrm{BE}$ have been carried out in the previous decade. Bile acid is a pathogenic factor of $\mathrm{BE}$ (3) and has been reported to promote its development through the upregulation of caudal type homeobox 2 expression (4). A recent study also demonstrated that the hedgehog pathway may contribute to the development of BE via regulation of the forkhead box A2 gene (5). Furthermore, expression levels of the intercellular adhesion molecule 1 and Kruppel like factor 4 genes were demonstrated to be significantly higher in $\mathrm{BE}$ tissues compared with control samples (6,7), whereas Rho-kinase gene expression was almost unchanged (8). Although these studies have made progress, most have only focused on disparate genes, which is not sufficient for providing an overall mechanism of BE development. Thus, the aim of the current study was to explore possible molecular mechanisms and potential biomarkers of BE using bioinformatic methods. Datasets were downloaded from the Gene Expression Omnibus (GEO) database (http://www.ncbi.nlm.nih.gov/geo/) and identified differentially expressed genes (DEGs) and differentially expressed microRNAs (DEmiRNAs) using the GEO2R program. Subsequently, DEGs were analyzed using functional and pathway enrichment analysis, and then their regulatory networks were constructed. Following prediction of the target genes of the DEmiRNAs, the target genes were integrated with the relevant DEGs to further identify the key genes involved in BE.

\section{Materials and methods}

Data resources. mRNA expression profiles (GSE26886, GSE13083 and GSE34619) and the miRNA expression profile 
of GSE20099 were downloaded from the GEO data repository. The dataset of GSE26886 contains $20 \mathrm{BE}$ samples and 19 normal esophageal samples. GSE13083 includes $7 \mathrm{BE}$ samples and 7 normal samples. GSE34619 consists of 10 BE samples and 8 normal samples. The miRNA expression dataset of GSE20099 contains 14 BE samples and 14 normal samples.

Differential expression analysis. GEO2R (http://www.ncbi.nl $\mathrm{m} . n i h . g o v / g e o / g e o 2 r /)$ is an online tool that performs comparisons on GEO datasets based on the GEOquery and Limma $\mathrm{R}$ packages (9). The BE group and normal group were selected, and the GEO2R program was subsequently applied for differential expression analysis. The genes and miRNAs that met the cut-off criteria of the adjusted P-value (adj. P) $<0.01$ and llog fold changel $>2$ were considered as DEGs and DEmiRNAs.

Functional and Kyoto Encyclopedia of Genes and Genomes (KEGG) pathway enrichment analysis. The Database for Annotation, Visualization and Integrated Discovery (DAVID; http://david.abcc.ncifcrf.gov/) is a gene functional enrichment program, providing a large series of functional annotation tools for researchers to decipher the biological implications behind huge amounts of genes. To understand the deeper biological meaning of DEGs, Gene Ontology (GO) and KEGG pathway enrichment analysis of identified DEGs was performed using DAVID (10). GO functional analysis consists of three categories: Biological process (BP), cellular component (CC) and molecular function (MF). $\mathrm{P}<0.05$ was set as the threshold value.

Protein-protein interaction (PPI) network. The Search Tool for the Retrieval of Interacting Genes/Proteins (STRING; http://string.embl.de/) is an online database containing known and predicted PPI networks. In this research, a PPI network of identified DEGs in all three datasets was identified using the STRING database (combined score $>0.4$ ) and subsequently visualized using Cytoscape (http://www.cytoscape.org/) software (version 3.4.0; The Cytoscape Consortium, San Diego, CA, USA) $(11,12)$. The Molecular Complex Detection (MCODE) program within Cytoscape was used to detect modules of the PPI network (13). The cut-off criteria were set as follows: Degree, $\geq 2$; node score, $\geq 0.2$; k-score, 0.2 ; maximum depth, 100. The function and pathway enrichment analysis of the identified modules was then performed using the DAVID database.

Prediction of miRNA targets. miRecords (http://c1.accurasc ience.com/ miRecords/) is an online program which integrates miRNA target predictions made by 11 established prediction databases (NBmiRTar, RNA22, MicroInspector, PicTar, PITA, RNAhybrid, and TargetScan, DIANA-microT, miRanda, MirTarget2 and miTarget) (14). In this study, potential targets of DEmiRNAs were identified by at least four databases.

\section{Results}

Differential expression analysis. Gene expression profiles GSE26886, GSE13083 and GSE34619 identified 2,112, 941 and 604 DEGs respectively, and 311 DEGs were identified across all three datasets (Fig. 1). Genes present in upregulated and

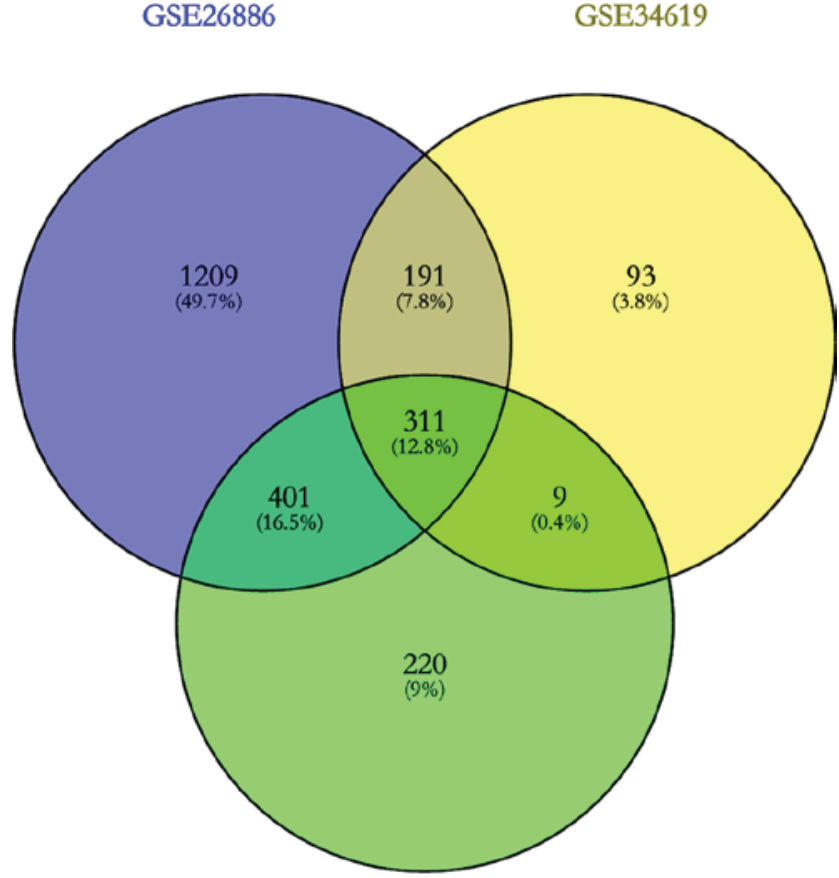

GSE13083

Figure 1. Identification of differentially expressed genes in gene expression profile datasets GSE26886, GSE13083 and GSE43619. Percentages displayed below the number of genes in each section indicates the proportion of genes in that section in regards to all three data sets.

downregulated groups in different datasets were excluded, and 311 DEGs still presented stable trends in at least two datasets, including 163 upregulated genes and 148 downregulated genes in BE samples compared with normal esophageal samples.

Functional and KEGG pathway enrichment analysis. The upregulated genes enriched in BP terms were mainly associated with digestion, tissue homeostasis and microvillus organization, upregulated genes enriched in CC terms were mainly associated with extracellular vesicles and upregulated genes enriched in MF terms were mainly associated with actin binding. Furthermore, KEGG pathway enrichment analysis demonstrated that the upregulated genes were mainly enriched in pancreatic secretion, metabolic pathways and salivary secretion pathways (Table I). The downregulated genes enriched in BP terms were mainly associated with epidermis development, keratinocyte differentiation and skin development, in CC terms the genes were mainly associated with extracellular regions and in MF terms the genes were mainly associated with receptor antagonist activity. In addition, KEGG pathway enrichment analysis revealed that the downregulated genes were mainly associated with cytochrome P450 drug metabolism and the amoebiasis pathway (Table II).

PPI network and modules analysis. A total of 204 nodes and 382 edges were obtained from the PPI network program. A degree of $>10$ was set as the cut-off criterion for hub gene identification. A total of 16 hub genes were identified: Keratin 16 (KRT16), cystic fibrosis transmembrane conductance regulator $(C F T R)$, involucrin $(I V L)$, protein kinase $\mathrm{C} \alpha(P R K C A)$, mucin 2, oligomeric mucus/gel-forming 
Table I. Functional and KEGG pathway enrichment analysis of upregulated genes in BE.

A, Biological process

\begin{tabular}{llrr}
\hline Term & \multicolumn{1}{c}{ Description } & Count & P-value \\
\hline GO:0007586 & Digestion & 13 & $3.91 \times 10^{-8}$ \\
GO:0001894 & Tissue homeostasis & 13 & $3.50 \times 10^{-7}$ \\
GO:0032528 & Microvillus organization & 6 & $9.49 \times 10^{-6}$ \\
GO:0030277 & Maintenance of gastrointestinal epithelium & 5 & $1.09 \times 10^{-5}$ \\
GO:0016266 & O-glycan processing & 7 & $1.68 \times 10^{-5}$ \\
\hline
\end{tabular}

B, Cellular component

\begin{tabular}{llll}
\hline Term & \multicolumn{1}{c}{ Description } & Count & P-value \\
\hline GO:1903561 & Extracellular vesicle & 73 & $1.24 \times 10^{-15}$ \\
GO:0043230 & Extracellular organelle & 73 & $1.26 \times 10^{-15}$ \\
GO:0070062 & Extracellular exosome & 72 & $3.61 \times 10^{-15}$ \\
GO:0031988 & Membrane-bounded vesicle & 78 & $1.55 \times 10^{-12}$ \\
GO:0044421 & Extracellular region part & 80 & $7.07 \times 10^{-12}$ \\
\hline
\end{tabular}

C, Molecular function

\begin{tabular}{llrr}
\hline Term & \multicolumn{1}{c}{ Description } & Count & P-value \\
\hline GO:0003779 & Actin binding & 14 & $6.49 \times 10^{-5}$ \\
GO:0022853 & Active ion transmembrane transporter activity & 7 & $4.72 \times 10^{-4}$ \\
GO:0050839 & Cell adhesion molecule binding & 13 & 0.001010 \\
GO:0008092 & Cytoskeletal protein binding & 18 & 0.001705 \\
GO:0022804 & Active transmembrane transporter activity & 11 & 0.001831
\end{tabular}

\section{D, KEGG}

\begin{tabular}{llrr}
\hline Term & \multicolumn{1}{c}{ Description } & Count & P-value \\
\hline hsa04972 & Pancreatic secretion & 8 & $4.20 \times 10^{-5}$ \\
hsa01100 & Metabolic pathways & 27 & $1.16 \times 10^{-4}$ \\
hsa04970 & Salivary secretion & 6 & 0.001815 \\
hsa05110 & Vibrio cholerae infection & 5 & 0.002050 \\
hsa04976 & Bile secretion & 5 & 0.005352
\end{tabular}

Count, the number of enriched genes in each term; GO, Gene Ontology; KEGG, Kyoto Encyclopedia of Genes and Genomes.

(MUC2), amyloid beta precursor protein $(A P P)$, cadherin 17 (CDH17), mucin 6, oligomeric mucus/gel-forming (MUC6), MET proto-oncogene, receptor tyrosine kinase $(M E T)$, envoplakin (EVPL), desmoglein 1 (DSGl), keratin 8 (KRT8), peroxisome proliferator activated receptor g $(P P A R G)$, prominin 1 (PROM1), cytochrome $\mathrm{P} 450$ family 2 subfamily $\mathrm{E}$ member 1 (CYP2E1) and filaggrin (FLG; Table III and Fig. 2). The three most significant modules were then selected from the PPI network using MCODE (Fig. 3). Notably, not all of the hub genes were present in these modules. Functional enrichment analysis of these modules was then performed. Genes in module A were mainly enriched in skin development, keratinocyte differentiation and keratinization. Genes in module B were predominantly enriched in O-glycan processing, mucin type $\mathrm{O}$-glycan biosynthesis and metabolic pathways. Finally, genes in module $\mathrm{C}$ were mainly enriched in homeostatic processes, pancreatic secretion and the bile secretion pathway (Table IV).

Integrated analysis of DEmiRNAs and paired DEGs. The miRNA expression profiles identified five DEmiRNAs, including two upregulated miRNAs [miRNA-215 (miR-215) and $m i R-192]$ and three downregulated miRNAs (miR-205, $m i R-203$ and $m i R-486-5 p)$ in BE samples compared with 
Table II. Functional and KEGG pathway enrichment analysis of downregulated genes in BE.

A, Biological process

\begin{tabular}{llrr}
\hline Term & \multicolumn{1}{c}{ Description } & Count & P-value \\
\hline GO:0008544 & Epidermis development & 21 & $3.50 \times 10^{-13}$ \\
GO:0030216 & Keratinocyte differentiation & 14 & $1.24 \times 10^{-11}$ \\
GO:0043588 & Skin development & 17 & $7.84 \times 10^{-11}$ \\
GO:0009913 & Epidermal cell differentiation & 15 & $1.71 \times 10^{-10}$ \\
GO:0031424 & Keratinization & 8 & $1.40 \times 10^{-7}$ \\
\hline
\end{tabular}

B, Cellular component

\begin{tabular}{llll}
\hline Term & \multicolumn{1}{c}{ Description } & Count & P-value \\
\hline GO:0005576 & Extracellular region & 73 & $5.14 \times 10^{-9}$ \\
GO:0044421 & Extracellular region part & 63 & $7.59 \times 10^{-8}$ \\
GO:0070062 & Extracellular exosome & 50 & $3.22 \times 10^{-7}$ \\
GO:1903561 & Extracellular vesicle & 50 & $3.76 \times 10^{-7}$ \\
GO:0043230 & Extracellular organelle & 50 & $3.81 \times 10^{-7}$ \\
\hline
\end{tabular}

C, Molecular function

\begin{tabular}{llrr}
\hline Term & \multicolumn{1}{c}{ Description } & Count & P-value \\
\hline GO:0048019 & Receptor antagonist activity & 3 & 0.002808 \\
GO:0030547 & Receptor inhibitor activity & 3 & 0.004791 \\
GO:0005149 & Interleukin-1 receptor binding & 3 & 0.006381 \\
GO:0005198 & Structural molecule activity & 14 & 0.010380 \\
GO:0005509 & Calcium ion binding & 13 & 0.013760
\end{tabular}

\section{D, KEGG}

\begin{tabular}{llll} 
Term & \multicolumn{1}{c}{ Description } & Count & P-value \\
\hline hsa00982 & Drug metabolism-cytochrome P450 & 4 & 0.013555 \\
hsa05146 & Amoebiasis & 4 & 0.042967
\end{tabular}

Count, the number of enriched genes in each term; GO, Gene Ontology; KEGG, Kyoto Encyclopedia of Genes and Genomes.

normal esophageal samples. Among them, $m i R-215$ and $m i R$-205 were the most significantly upregulated and downregulated miRNAs, respectively. In addition, predicted targets of DEmiRNAs were obtained from miRecords database. Considering the fact that an inverse association exists between the expression of miRNA and its target mRNA, DEmiRNAs with target genes that were identified as DEGs were selected. Remarkably, 33 pairs of DEmiRNAs and DEGs with an inverse relationship of expression met this criterion. Among these target genes, PRKCA was the target of two downregulated miRNAs ( $m i R-203$ and $m i R-205)$, while epiregulin (EREG) was the target of two upregulated miRNAs (miR-215 and miR-192). Furthermore, two hub genes (PRKCA and CDH17) were predicted as targets of $m i R-203$ (Table V).

\section{Discussion}

$\mathrm{BE}$ is the precursor lesion of EAC and is still not completely understood (2). Therefore, the aim of the current study was to provide an overall view of the molecular mechanism and biomarkers in BE. In the present research, a total of 311 DEGs and five DEmiRNAs were identified from GEO databases using the GEO2R program. The functional analysis of 163 upregulated DEGs demonstrated these genes were mainly associated with digestion, tissue homeostasis and microvillus organization, while the functional analysis of 148 downregulated DEGs demonstrated that they were associated with epidermis development, keratinocyte differentiation and skin development. Following construction of the PPI network, 16 hub genes were identified. The target genes of DEmiRNAs 
Table III. Identified hub genes in the protein-protein interaction network.

\begin{tabular}{|c|c|c|c|c|c|c|}
\hline \multirow[b]{2}{*}{ Gene } & \multicolumn{2}{|c|}{ GSE26686 } & \multicolumn{2}{|c|}{ GSE13083 } & \multicolumn{2}{|c|}{ GSE34619 } \\
\hline & $\log \mathrm{FC}$ & adj. $P$ & $\log \mathrm{FC}$ & adj. $P$ & $\log \mathrm{FC}$ & adj. $P$ \\
\hline KRT16 & -4.62 & $3.33 \times 10^{-4}$ & -5.07 & $5.63 \times 10^{-3}$ & -3.52 & $2.61 \times 10^{-5}$ \\
\hline CFTR & 2.35 & $8.6 \times 10^{-5}$ & 5.33 & $1.08 \times 10^{-6}$ & 4.31 & $1.61 \times 10^{-11}$ \\
\hline$I V L$ & -6.29 & $2.10 \times 10^{-6}$ & -4.35 & $2.53 \times 10^{-4}$ & -4.27 & $1.79 \times 10^{-11}$ \\
\hline$P R K C A$ & 2.79 & $6.77 \times 10^{-7}$ & 3.31 & $2.07 \times 10^{-5}$ & 2.36 & $2.63 \times 10^{-11}$ \\
\hline$M U C 2$ & 4.21 & $4.34 \times 10^{-5}$ & 6.72 & $3.16 \times 10^{-6}$ & 2.20 & $2.77 \times 10^{-3}$ \\
\hline$A P P$ & 2.41 & $4.04 \times 10^{-6}$ & 3.56 & $1.08 \times 10^{-5}$ & 2.09 & $2.31 \times 10^{-8}$ \\
\hline$C D H 17$ & 5.58 & $8.00 \times 10^{-5}$ & 7.36 & $5.66 \times 10^{-8}$ & 4.31 & $2.77 \times 10^{-4}$ \\
\hline MUC6 & 6.11 & $1.02 \times 10^{-6}$ & 3.10 & $7.45 \times 10^{-3}$ & 5.94 & $1.46 \times 10^{-11}$ \\
\hline$M E T$ & 2.60 & $7.40 \times 10^{-9}$ & 4.21 & $1.27 \times 10^{-6}$ & 2.32 & $9.04 \times 10^{-9}$ \\
\hline$E V P L$ & -3.66 & $2.23 \times 10^{-7}$ & -3.55 & $6.81 \times 10^{-4}$ & -2.10 & $7.23 \times 10^{-12}$ \\
\hline$D S G 1$ & -3.85 & $2.13 \times 10^{-3}$ & -4.84 & $4.51 \times 10^{-5}$ & -5.48 & $1.06 \times 10^{-7}$ \\
\hline KRT8 & 5.73 & $5.13 \times 10^{-15}$ & 6.42 & $2.18 \times 10^{-9}$ & 4.01 & $3.50 \times 10^{-11}$ \\
\hline PPARG & 4.56 & $2.02 \times 10^{-12}$ & 3.37 & $1.48 \times 10^{-6}$ & 3.02 & $1.66 \times 10^{-11}$ \\
\hline PROMI & 8.47 & $1.02 \times 10^{-17}$ & 7.37 & $4.24 \times 10^{-11}$ & 5.44 & $2.03 \times 10^{-14}$ \\
\hline CYP2E1 & -4.34 & $1.66 \times 10^{-7}$ & -4.88 & $1.43 \times 10^{-6}$ & -2.06 & $1.59 \times 10^{-8}$ \\
\hline$F L G$ & -4.40 & $5.87 \times 10^{-4}$ & -6.16 & $3.09 \times 10^{-5}$ & -4.96 & $1.14 \times 10^{-10}$ \\
\hline
\end{tabular}

FC, fold change; adj. P, adjusted P-value.

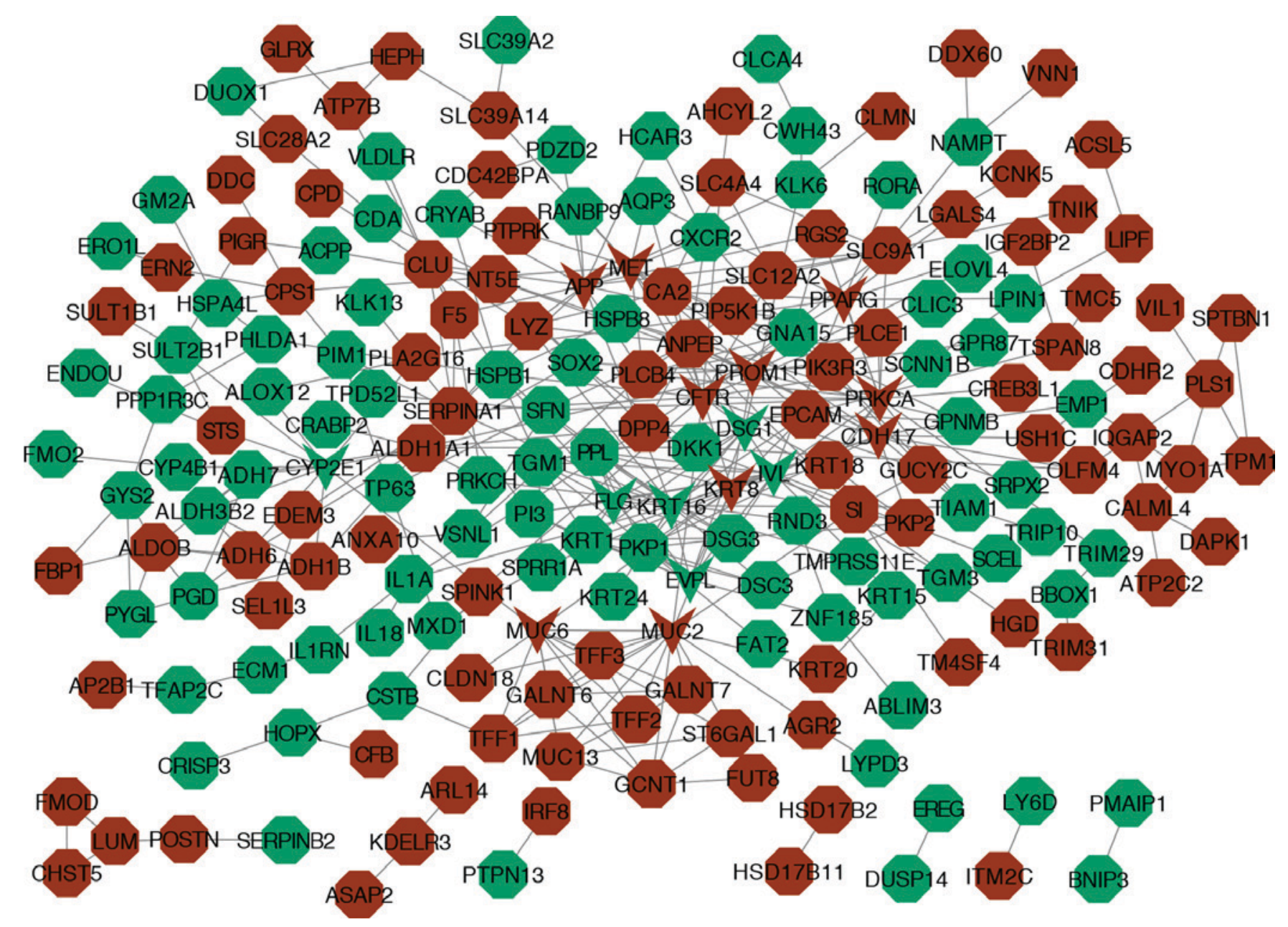

Figure 2. The protein-protein network of identified differentially expressed genes. Red nodes and green nodes indicate upregulated genes and downregulated genes, respectively; V-shape nodes represent the identified hub genes. 
Table IV. Functional and KEGG pathway enrichment analysis of gene modules.

A, Module A

\begin{tabular}{llrr}
\hline Term & \multicolumn{1}{c}{ Description } & Count & P-value \\
\hline GO:0043588 & Skin development & 7 & $2.13 \times 10^{-10}$ \\
GO:0030216 & Keratinocyte differentiation & 6 & $9.43 \times 10^{-10}$ \\
GO:0031424 & Keratinization & 5 & $4.99 \times 10^{-9}$ \\
GO:0009913 & Epidermal cell differentiation & 6 & $7.20 \times 10^{-9}$ \\
GO:0008544 & Epidermis development & 6 & $9.84 \times 10^{-8}$ \\
\hline
\end{tabular}

B, Module B

\begin{tabular}{llrr}
\hline Term & \multicolumn{1}{c}{ Description } & Count & P-value \\
\hline GO:0016266 & O-glycan processing & 7 & $1.41 \times 10^{-13}$ \\
GO:0006493 & Protein O-linked glycosylation & 7 & $4.24 \times 10^{-12}$ \\
GO:0005975 & Carbohydrate metabolic process & 9 & $1.91 \times 10^{-10}$ \\
GO:0043413 & Macromolecule glycosylation & 7 & $1.65 \times 10^{-9}$ \\
GO:0006486 & Protein glycosylation & 7 & $1.65 \times 10^{-9}$ \\
hsa00512 & Mucin type O-Glycan biosynthesis & 3 & $1.16 \times 10^{-4}$ \\
hsa01100 & Metabolic pathways & 4 & 0.019425 \\
\hline
\end{tabular}

C, Module C

\begin{tabular}{llrr}
\hline Term & \multicolumn{1}{c}{ Description } & Count & P-value \\
\hline GO:0042592 & Homeostatic process & 10 & $5.77 \times 10^{-6}$ \\
GO:0019725 & Cellular homeostasis & 8 & $6.83 \times 10^{-6}$ \\
GO:0030003 & Cellular cation homeostasis & 7 & $1.09 \times 10^{-5}$ \\
GO:0006873 & Cellular ion homeostasis & 7 & $1.24 \times 10^{-5}$ \\
GO:0055080 & Cation homeostasis & 7 & $2.09 \times 10^{-5}$ \\
hsa04972 & Pancreatic secretion & 6 & $1.07 \times 10^{-6}$ \\
hsa04976 & Bile secretion & 4 & $3.98 \times 10^{-4}$ \\
hsa04971 & Gastric acid secretion & 4 & $4.70 \times 10^{-4}$ \\
hsa04970 & Salivary secretion & 4 & $7.60 \times 10^{-4}$ \\
hsa04070 & Phosphatidylinositol signaling system & 4 & 0.001112
\end{tabular}

Count, the number of enriched genes in a certain term; GO, Gene Ontology; KEGG, Kyoto Encyclopedia of Genes and Genomes.

A

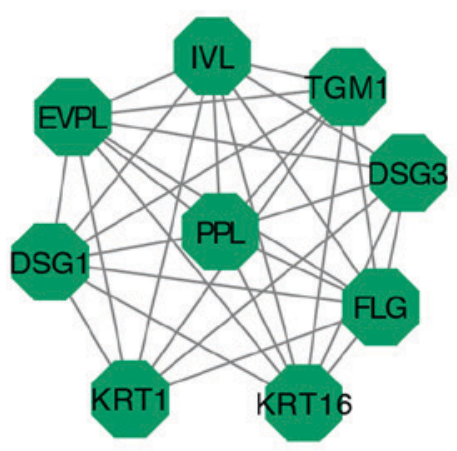

B

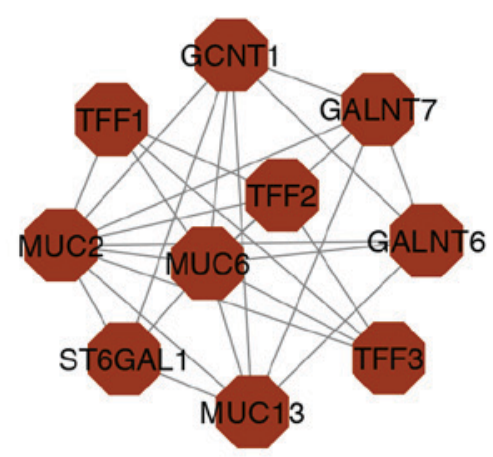

C

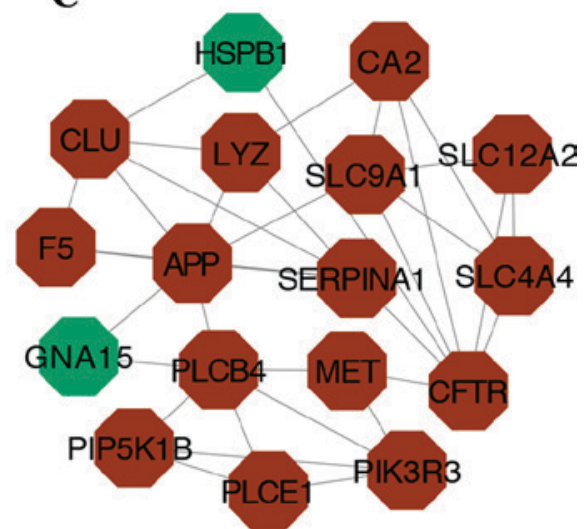

Figure 3. Top three modules identified from the protein-protein interaction network. (A) Module A, (B) module B and (C) module C. Red nodes and green nodes indicate upregulated genes and downregulated genes, respectively. 
Table V. Differentially expressed miRs and their paired DEGs in Barret's esophagus.

\begin{tabular}{lcrl}
\hline miR & $\begin{array}{c}\text { Adjusted } \\
\text { P-value }\end{array}$ & $\begin{array}{c}\text { Log fold } \\
\text { change }\end{array}$ & Paired DEGs \\
\hline hsa-miR-215 & $1.05 \times 10^{-11}$ & 5.972098 & EREG \\
hsa-miR-192 & $1.89 \times 10^{-6}$ & 2.665345 & EREG \\
hsa-miR-205 & $1.76 \times 10^{-3}$ & -3.301436 & $P R K C A, A B H D 2$, \\
& & & KDELR3, DPP4, \\
& & & HSDI7B11, MLPH \\
hsa-miR-203 & $3.04 \times 10^{-3}$ & -2.784608 & PRKCA, CDH17, \\
& & & CPS1, TOX3, \\
& & & CPD, STS, PIGR, \\
& & & FUT8, KRT20, \\
& & & PBLD \\
& & & TM4SF20 \\
hsa-miR-486-5p & $2.77 \times 10^{-4}$ & -2.506280 &
\end{tabular}

miR, microRNA; DEGs, differentially expressed genes.

were subsequently integrated with the DEGs, leading to the identification of three key genes that may be regulated by miRNAs.

KEGG pathway enrichment analysis revealed that the upregulated DEGs were mainly associated with pancreatic secretion, metabolic pathways, salivary secretion, vibrio cholera infection and bile secretion. Many clinical studies have focused on these pathways. Greer et al (15) reported that the insulin/insulin-like growth factor (IGF) pathway, which is associated with pancreatic secretion, may have a role in the development of BE. Regarding the importance of metabolic pathways, accumulating evidence suggests that obesity is a central risk factor for BE (16-18). Furthermore, high serum leptin and adiponectin have been shown to be associated with the development of BE (19-21).

As mentioned previously, BE is a columnar metaplasia in response to GERD. GERD can be a result of the impairment of the anti-reflux barrier, which in turn is associated with salivary secretion function $(22,23)$. In addition, bile exposure has confirmed effects in the development of BE $(24,25)$. Reveiller et al (26) demonstrated that the expression of squamous differentiation genes such as $I V L$ and $D S G 1$ was inhibited by bile exposure in human esophageal epithelial cells. Notably, IVL and DSGI are among our identified hub genes.

The downregulated DEGs identified were mainly associated with the cytochrome P450 drug metabolism pathway. Supporting this finding, a study previously demonstrated that cytochrome P450 family 1 subfamily A member 2 (CYP1A2), CYP3A4 and CYP2E1 were highly expressed in regions of active cell proliferation in BE (27).

The results of the functional and pathway analysis performed on the gene modules are largely similar to the DEGs mentioned above. The module enrichment analysis also indicated that skin development, mucin type O-glycan biosynthesis and homeostatic processes were associated with the development of BE. The normal esophageal epithelium is made up of squamous epithelium, which also constitutes the skin. The genes associated skin development were all downregulated. Clinical manifestation of this change in gene expression is observed in the replacement of squamous epithelium with columnar epithelium that is typical of BE (1). Notably, genes associated with mucin type O-glycan in module B were all upregulated in BE samples, which was correlated with the degree of intestinal metaplasia in BE. Mucin type O-glycan has been reported to have important roles in intestinal homeostasis (28). A previous study indicated that dysfunction of homeostatic processes may be vital in the development of $\mathrm{BE}$ (29). Therefore, a focus on these pathways may provide us with greater knowledge of BE development.

miRNAs are a group of endogenous non-coding RNA molecules that act as negative regulators in post-transcriptional gene regulation. Recent studies reported that aberrant miRNA expression is associated with the development of $\mathrm{BE}(30,31)$. The current study found five differentially expressed miRNAs, including two upregulated DEmiRNAs and three downregulated DEmiRNAs. Among them, miR-215 and miR-205 were the most significantly upregulated and downregulated DEmiRNAs, respectively. Other studies have also confirmed the aberrant expression of these two miRNAs in BE and EAC $(32,33) . m i R-215$ is involved in many diseases, including diabetic nephropathy, ovarian cancer, non-small cell lung cancer and multiple carcinomas. miR-215 commonly acts as a tumor suppressor by promoting apoptosis (34-36). miR-205 also has an effect in various diseases and may act as a prognostic marker for many malignant neoplasms $(37,38)$. This integrated study identified that two hub genes (PRKCA and CDH17) were target genes of $m i R-203$, and $E R E G$ was a target of two upregulated miRNAs (miR-215 and miR-192).

As a member of the serine/threonine-specific protein kinase family, PRKCA is involved in various biological processes, including the innate immune response, angiogenesis, insulin secretion, cell proliferation, adhesion and migration. In addition, PRKCA was also identified to be involved in several pathways, including the mitogen-activated protein kinase (MAPK) pathway, phosphoinositide 3-kinase (PI3K)-Akt pathway and Wnt pathway. Previous studies have demonstrated that the MAPK and PI3K pathways are involved in the proliferation of BE-associated EAC (39), while the insulin/IGF and Wnt pathways have important roles in BE development $(15,40)$.

$\mathrm{CDH17}$, which is located on chromosome 8, encodes liver-intestinal cadherin (LI-cadherin), which is involved in cell adhesion and oligopeptide transport. LI-cadherin is localized at cell junctions and is selectively expressed in enterocytes and intestinal goblet cells, but not in the esophagus (41). Multiple studies have reported that aberrant LI-cadherin expression is a sensitive marker for detection of various metaplastic diseases such as gastric intestinal metaplasia and BE $(42,43)$.

$E R E G$, located on chromosome 4, encodes the epiregulin protein, which is involved in various biological processes, including the innate immune response, cytokine production, wound healing and epithelial cell proliferation. A previous study also demonstrated that epiregulin regulates the differentiation of airway epithelial cells through the epidermal growth factor receptor pathway (44). Furthermore, epiregulin has roles in a series of other malignant diseases as well as 
esophageal cancer, and can be used as biomarker in certain diseases (45-47). Taken together, this data indicates that the genes PRCKA, CDH17 and EREG may be involved in the development of $\mathrm{BE}$ through various pathways.

There are certain limitations to the present study. Due to the fact that there is limited research available on the pathways involved in BE, the identified KEGG pathways could not be discussed in detail. Additionally, excluding miRNA data, the datasets together provided only $37 \mathrm{BE}$ samples and 34 control samples. Samples obtained from different platforms may also suffer from some bias. The high level of redundancy in the GO database was also a limiting factor in the study. Further research should focus on methods to evaluate possible bias that may arise in this type of research.

In conclusion, the present study performed a comprehensive bioinformatics analysis of identified DEGs to identify potential pathways and biomarkers involved in the development of BE. In the current research, 311 DEGs and 5 DEmiRNAs were identified. Following integration of the DEGs with any corresponding DEmiRNAs, three key genes (PRKCA, CDH17 and $E R E G$ ) were identified that may be associated with BE. This research provides a novel insight into molecular mechanisms that may underlie the development of BE. However, biochemical analysis and further research are still necessary to validate these results.

\section{Acknowledgements}

The present study is supported by The National Natural Science Foundation of China (grant no. 81370485).

\section{References}

1. Falk GW, Jacobson BC, Riddell RH, Rubenstein JH, El-Zimaity H, Drewes AM, Roark KS, Sontag SJ, Schnell TG, Leya J, et al: Barrett's esophagus: Prevalence-incidence and etiology-origins. Ann N Y Acad Sci 1232: 1-17, 2011.

2. Solaymani-Dodaran M, Logan RF, West J, Card T and Coupland C: Risk of oesophageal cancer in Barrett's oesophagus and gastro-oesophageal reflux. Gut 53: 1070-1074, 2004.

3. Tatsugami $M$, Ito $M$, Tanaka $S$, Yoshihara $M$, Matsui $H$, Haruma K and Chayama K: Bile acid promotes intestinal metaplasia and gastric carcinogenesis. Cancer Epidemiol Biomarkers Prev 21: 2101-2107, 2012.

4. $\mathrm{Hu}$ Y, Williams VA, Gellersen O, Jones C, Watson TJ and Peters JH: The pathogenesis of Barrett's esophagus: Secondary bile acids upregulate intestinal differentiation factor CDX2 expression in esophageal cells. J Gastrointest Surg 11: 827-834, 2007.

5. Wang DH, Tiwari A, Kim ME, Clemons NJ, Regmi NL, Hodges WA, Berman DM, Montgomery EA, Watkins DN, Zhang X, et al: Hedgehog signaling regulates FOXA2 in esophageal embryogenesis and Barrett's metaplasia. J Clin Invest 124 3767-3780, 2014

6. Alasehirli B, Oğuz E, Oksuzler E, Koruk I, Oztuzcu S, Ozkara E, Karakok M, Erbagcı AB and Demiryurek AT: Investigation of intercellular adhesion molecules (ICAMs) gene expressions in patients with Barrett's esophagus. Tumour Biol 35: 4907-4912, 2014.

7. Kazumori H, Ishihara S, Takahashi Y, Amano Y and Kinoshita Y: Roles of Kruppel-like factor 4 in oesophageal epithelial cells in Barrett's epithelium development. Gut 60: 608-617, 2011.

8. Demiryürek S, Koruk I, Bozdag Z, Ozkara E, Kaplan DS, Oztuzcu S, Cetinkaya A, Alasehirli B and Demiryürek AT: Investigation of the esophageal Rho-kinase expression in patients with Barrett's esophagus. Ultrastruct Pathol 37: 284-289, 2013.

9. Barrett T, Wilhite SE, Ledoux P, Evangelista C, Kim IF, Tomashevsky M, Marshall KA, Phillippy KH, Sherman PM, Holko M, et al: NCBI GEO: Archive for functional genomics data sets-update. Nucleic Acids Res 41 (Database Issue): D991-D995, 2013.
10. Huang da W, Sherman BT and Lempicki RA: Systematic and integrative analysis of large gene lists using DAVID bioinformatics resources. Nat Protoc 4: 44-57, 2009.

11. Szklarczyk D, Franceschini A, Wyder S, Forslund K, Heller D, Huerta-Cepas J, Simonovic M, Roth A, Santos A, Tsafou KP, et al: STRING v10: Protein-protein interaction networks, integrated over the tree of life. Nucleic Acids Res 43 (Database Issue): D447-D452, 2015.

12. Shannon P, Markiel A, Ozier O, Baliga NS, Wang JT, Ramage D, Amin N, Schwikowski B and Ideker T: Cytoscape: A software environment for integrated models of biomolecular interaction networks. Genome Res 13: 2498-2504, 2003.

13. Bader GD and Hogue CW: An automated method for finding molecular complexes in large protein interaction networks. BMC Bioinformatics 4: 2, 2003.

14. Xiao F, Zuo Z, Cai G, Kang S, Gao X and Li T: miRecords: An integrated resource for microRNA-target interactions. Nucleic Acids Res 37 (Database Issue): D105-D110, 2009.

15. Greer KB, Thompson CL, Brenner L, Bednarchik B, Dawson D, Willis J, Grady WM, Falk GW, Cooper GS, Li L and Chak A: Association of insulin and insulin-like growth factors with Barrett's oesophagus. Gut 61: 665-672, 2012.

16. Lee SW, Lien HC, Chang CS, Lee TY, Peng YC and Yeh HZ: Association of metabolic syndrome with erosive esophagitis and Barrett's esophagus in a Chinese population. J Chin Med Assoc 80: 15-18, 2017.

17. Crews NR, Johnson ML, Schleck CD, Enders FT, Wongkeesong LM, Wang KK, Katzka DA and Iyer PG: Prevalence and predictors of gastroesophageal reflux complications in community subjects. Dig Dis Sci 61: 3221-3228, 2016.

18. Singh S, Sharma AN, Murad MH, Buttar NS, El-Serag HB, Katzka DA and Iyer PG: Central adiposity is associated with increased risk of esophageal inflammation, metaplasia, and adenocarcinoma: A systematic review and meta-analysis. Clin Gastroenterol Hepatol 11: 1399-1412.e7, 2013.

19. Kendall BJ, Macdonald GA, Hayward NK, Prins JB, Brown I, Walker N, Pandeya N, Green AC, Webb PM and Whiteman DC; Study of Digestive Health: Leptin and the risk of Barrett's oesophagus. Gut 57: 448-454, 2008.

20. Thompson OM, Beresford SA, Kirk EA, Bronner MP and Vaughan TL: Serum leptin and adiponectin levels and risk of Barrett's esophagus and intestinal metaplasia of the gastroesophageal junction. Obesity (Silver Spring) 18: 2204-2211, 2010.

21. Rubenstein JH, Morgenstern H, McConell D, Scheiman JM, Schoenfeld P, Appelman H, McMahon LF Jr, Kao JY, Metko V, Zhang $\mathrm{M}$ and Inadomi JM: Associations of diabetes mellitus, insulin, leptin, and ghrelin with gastroesophageal reflux and Barrett's esophagus. Gastroenterology 145: 1237-1244.e1-5, 2013.

22. Cassiani RA, Mota GA, Aprile LR and Dantas RO: Saliva transit in patients with gastroesophageal reflux disease. Dis Esophagus 28: 673-677, 2015.

23. Skoczylas T, Yandrapu H, Poplawski C, Asadi M, Wallner G and Sarosiek J: Salivary bicarbonate as a major factor in the prevention of upper esophageal mucosal injury in gastroesophageal reflux disease. Dig Dis Sci 59: 2411-2416, 2014.

24. Sun D, Wang X, Gai Z, Song X, Jia X and Tian H: Bile acids but not acidic acids induce Barrett's esophagus. Int J Clin Exp Pathol 8: 1384-1392, 2015.

25. Takahashi Y, Amano Y, Yuki T, Mishima Y, Tamagawa Y, Uno G, Ishimura N, Sato S, Ishihara S and Kinoshita Y: Impact of the composition of gastric reflux bile acids on Barrett's oesophagus. Dig Liver Dis 43: 692-697, 2011.

26. Reveiller M, Ghatak S, Toia L, Kalatskaya I, Stein L, D'Souza M, Zhou Z, Bandla S, Gooding WE, Godfrey TE and Peters JH: Bile exposure inhibits expression of squamous differentiation genes in human esophageal epithelial cells. Ann Surg 255: 1113-1120, 2012.

27. Hughes SJ, Morse MA, Weghorst CM, Kim H, Watkins PB, Guengerich FP, Orringer MB and Beer DG: Cytochromes P450 are expressed in proliferating cells in Barrett's metaplasia. Neoplasia 1: 145-153, 1999.

28. Bergstrom KS and Xia L: Mucin-type O-glycans and their roles in intestinal homeostasis. Glycobiology 23: 1026-1037, 2013.

29. Rosekrans SL, Baan B, Muncan V and van den Brink GR: Esophageal development and epithelial homeostasis. Am J Physiol Gastrointest Liver Physiol 309: G216-G228, 2015.

30. Cabibi D, Caruso S, Bazan V, Castiglia M, Bronte G, Ingrao S, Fanale D, Cangemi A, Calò V, Listì A, et al: Analysis of tissue and circulating microRNA expression during metaplastic transformation of the esophagus. Oncotarget 7: 47821-47830, 2016. 
31. Bus P, Kestens C, Ten Kate FJ, Peters W, Drenth JP, Roodhart JM, Siersema PD and van Baal JW: Profiling of circulating microRNAs in patients with Barrett's esophagus and esophageal adenocarcinoma. J Gastroenterol 51: 560-570, 2016.

32. Slaby O, Srovnal J, Radova L, Gregar J, Juracek J, Luzna P, Svoboda M, Hajduch M and Ehrmann J: Dynamic changes in microRNA expression profiles reflect progression of Barrett's esophagus to esophageal adenocarcinoma. Carcinogenesis 36 : 521-527, 2015.

33. Wijnhoven BP, Hussey DJ, Watson DI, Tsykin A, Smith CM and Michael MZ; South Australian Oesophageal Research Group: MicroRNA profiling of Barrett's oesophagus and oesophageal adenocarcinoma. Br J Surg 97: 853-861, 2010.

34. Jia Y, Guan M, Zheng Z, Zhang Q, Tang C, Xu W, Xiao Z, Wang $L$ and Xue $Y$ : miRNAs in urine extracellular vesicles as predictors of early-stage diabetic nephropathy. J Diabetes Res 2016: 7932765, 2016.

35. Ge G, Zhang W, Niu L, Yan Y, Ren Y and Zou Y: miR-215 functions as a tumor suppressor in epithelial ovarian cancer through regulation of the X-chromosome-linked inhibitor of apoptosis. Oncol Rep 35: 1816-1822, 2016.

36. Hou Y, Zhen J, Xu X, Zhen K, Zhu B, Pan R and Zhao C: miR-215 functions as a tumor suppressor and directly targets ZEB2 in human non-small cell lung cancer. Oncol Lett 10: 1985-1992, 2015.

37. Xu H, Yao Y, Meng F, Qian X, Jiang X, Li X, Gao Z and Gao L: Predictive Value of Serum miR-10b, miR-29c, and miR-205 as promising biomarkers in esophageal squamous cell carcinoma screening. Medicine (Baltimore) 94: e1558, 2015.

38. Yue X, Lan F, Hu M, Pan Q, Wang Q and Wang J: Downregulation of serum microRNA-205 as a potential diagnostic and prognostic biomarker for human glioma. J Neurosurg 124: 122-128, 2016

39. Vona-Davis L, Frankenberry K, Cunningham C, Riggs DR, Jackson BJ, Szwerc MF and McFadden DW: MAPK and PI3K inhibition reduces proliferation of Barrett's adenocarcinoma in vitro. J Surg Res 127: 53-58, 2005.
40. Chen X, Jiang K, Fan Z, Liu Z, Zhang P, Zheng L, Peng N, Tong J and Ji G: Aberrant expression of Wnt and Notch signal pathways in Barrett's esophagus. Clin Res Hepatol Gastroenterol 36: 473-483, 2012.

41. Gessner R and Tauber R: Intestinal cell adhesion molecules. Liver-intestine cadherin. Ann N Y Acad Sci 915: 136-143, 2000.

42. Mokrowiecka A, Zonnur S, Veits L, Musial J, Kordek R Lochowski M, Kozak J, Malecka-Panas E, Vieth M and Hartmann A: Liver-intestine-cadherin is a sensitive marker of intestinal differentiation during Barrett's carcinogenesis. Dig Dis Sci 58: 699-705, 2013

43. GrötzingerC,Kneifel J, Patschan D, Schnoy N, Anagnostopoulos I, Faiss S, Tauber R, Wiedenmann B and Gessner R: LI-cadherin: A marker of gastric metaplasia and neoplasia. Gut 49: 73-81, 2001.

44. Vermeer PD, Panko L, Karp P, Lee JH and Zabner J: Differentiation of human airway epithelia is dependent on erbB2. Am J Physiol Lung Cell Mol Physiol 291: L175-L180, 2006.

45. Sun L, Pan J, Yu L, Liu H, Shu X, Sun L, Lou J, Yang Z and Ran Y: Tumor endothelial cells promote metastasis and cancer stem cell-like phenotype through elevated Epiregulin in esophageal cancer. Am J Cancer Res 6: 2277-2288, 2016.

46. Amsterdam A, Shezen E, Raanan C, Slilat Y, Ben-Arie A, Prus D and Schreiber L: Epiregulin as a marker for the initial steps of ovarian cancer development. Int J Oncol 39: 1165-1172, 2011.

47. Watanabe T, Kobunai T, Yamamoto Y, Kanazawa T, Konishi T, Tanaka T, Matsuda K, Ishihara S, Nozawa K, Eshima K, et al: Prediction of liver metastasis after colorectal cancer using reverse transcription-polymerase chain reaction analysis of 10 genes. Eur J Cancer 46: 2119-2126, 2010.

This work is licensed under a Creative Commons Attribution-NonCommercial-NoDerivatives 4.0 International (CC BY-NC-ND 4.0) License. 